\title{
Embarazo y enfermedad inflamatoria intestinal, una combinación especial: a propósito de un caso y revisión de la literatura
}

\section{A special combination of pregnancy and inflammatory bowel disease: case report and literature review}

\author{
Viviana Parra Izquierdo ${ }^{1 *}$, Carolina Pavez Ovalle ${ }^{2}$, Alan Ovalle ${ }^{3}$, Carlos Espinosa ${ }^{4}$, Valeria Costa ${ }^{4}$, Gerardo Puentes ${ }^{4}$, Albis Hani5
}

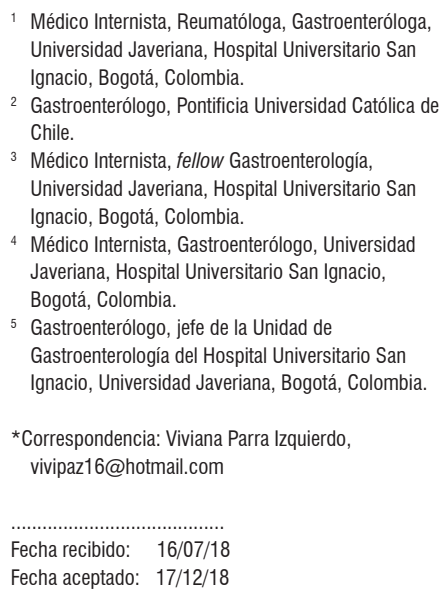

${ }^{3}$ Médico Internista, fellow Gastroenterología, Universidad Javeriana, Hospital Universitario San Ignacio, Bogotá, Colombia.

${ }^{4}$ Médico Internista, Gastroenterólogo, Universidad Javeriana, Hospital Universitario San Ignacio, Bogotá, Colombia.

${ }_{5}$ Gastroenterólogo, jefe de la Unidad de Gastroenterología del Hospital Universitario San Ignacio, Universidad Javeriana, Bogotá, Colombia.

${ }^{*}$ Correspondencia: Viviana Parra Izquierdo, vivipaz16@hotmail.com

Fecha recibido: $\quad 16 / 07 / 18$

Fecha aceptado: 17/12/18

\begin{abstract}
Resumen
La enfermedad inflamatoria intestinal (EII) comprende un espectro de enfermedades crónicas inmunomediadas que afectan el tracto gastrointestinal, con inicio típico durante el comienzo de la edad adulta. Esta enfermedad ha tenido un incremento de su incidencia a nivel mundial, teniendo en Norteamérica una mayor incidencia. En relación con la enfermedad de Crohn (EC) (20,2 por cada 100000 personas/año) y en Europa una incidencia de colitis ulcerativa (CU) (24,3 por 100000 personas/año), considerando que no es una enfermedad curable, la remisión de la misma es el principal objetivo del manejo. Muchas mujeres se encuentran afectadas por Ell durante distintos estadios de su vida, incluido durante la vida reproductiva, el embarazo y la menopausia. Es por lo anterior que la forma en la que se maneje la enfermedad en mujeres en edad reproductiva puede afectar el curso de la Ell. Son muy relevantes las estrategias de tratamiento y mantenimiento de la salud, para las pacientes con deseo de paridad, la remisión de la enfermedad es muy importante en el momento de la concepción y durante todo el embarazo, para asegurar adecuados resultados tanto para la madre como para el feto. Es bien conocido que la enfermedad activa durante el momento de la concepción y el embarazo se asocia con desenlaces adversos. Además, la enfermedad perianal activa es indicación de parto por cesárea, lo que resulta en un mayor riesgo de cirugía intestinal y complicaciones en el posoperatorio. A continuación, presentamos un caso.
\end{abstract}

\section{Palabras clave}

Enfermedad inflamatoria intestinal, enfermedad de Crohn, colitis ulcerativa, embarazo.

\begin{abstract}
Inflammatory bowel disease (IBD) comprises a spectrum of chronic immune-mediated diseases that affect the gastrointestinal tract. Onset typical occurs in adulthood. Its incidence is increasing everywhere, the highest incidence of Crohn's disease of 20.2 per 100,000 people/year is in North America while the incidence of ulcerative colitis is 24.3 per 100,000 people/year in Europe. Since it is not curable, the remission is the main objective of management. Many women are affected by IBD at different stages of their lives, including during reproductive life, pregnancy and menopause, so the way the disease is managed in reproductive age women can affect IBD's course. Treatment and maintenance strategies are very relevant. For patients with a desire to have children, disease remission is very important from conception through pregnancy to birth to ensure adequate results for both mother and fetus. It is well known that active disease during conception and pregnancy is associated with adverse outcomes of pregnancy. In addition, active perianal disease is an indication for cesarean delivery which entails increased risk of bowel surgery and complications in the postoperative period. We present a case of IBD during pregnancy.
\end{abstract}

\section{Keywords}

Inflammatory bowel disease, Crohn's disease, ulcerative colitis, pregnancy. 
Mujer de 32 años con cuadro de diarrea crónica con mucosidad y sangre asociado con dolor abdominal tipo cólico intermitente, en manejo con múltiples esquemas antibióticos y mejoría parcial. 2 años antes del inicio del cuadro clínico cursó con un embarazo, el cual a las 26 semanas fue interrumpido por óbito fetal. De forma concomitante presenta exacerbación de los síntomas gastrointestinales. El producto del embarazo presentaba malformaciones fetales dadas por sobrecrecimiento de extremidad, microsomía hemifacial e hidrops. Se realiza colonoscopia, en la cual se evidencia colitis ulcerativa (CU) severa con compromiso del colon izquierdo, por lo que se realiza diagnóstico de enfermedad inflamatoria intestinal (EII) y se inicia manejo con esteroides $40 \mathrm{mg} /$ día y mesalazina $4 \mathrm{~g}$ vía oral/día. La paciente mejora su estado clínico con el uso de esteroides, pero sin lograr su destete, por lo que se considera enfermedad corticodependiente y se inicia terapia biológica tipo antiTNF (infliximab) y tiopurinas (azatioprina $2,5 \mathrm{mg} / \mathrm{kg} /$ día).

Durante la evolución clínica, la paciente presenta proceso infeccioso severo, por lo cual requiere suspensión de la terapia biológica y se continúa manejo con azatioprina y mesalazina. A la edad de 37 años presenta nueva hospitalización con exacerbación severa de la enfermedad tanto clínica como endoscópica, desencadenada por mala adherencia al tratamiento instaurado. Requiere nuevamente manejo con esteroides y, por criterios de corticorresistencia, se decide reiniciar la terapia con antiTNF (infliximab) y se continúa con azatioprina y mesalazina. La paciente, al estar con buen control de la enfermedad, consulta por gestación en curso de 5 semanas. Se le indica la continuación de la terapia biológica y se le suspende la tiopurina; sin embargo, la paciente decide por su voluntad suspender todo tipo de medicación, a pesar del consejo médico. El embarazo transcurre con normalidad, sin ninguna complicación, presenta parto por cesárea con feto vivo con bajo peso al nacer, sin otras complicaciones. Durante el posparto mediato, la paciente presenta exacerbación de la enfermedad intestinal, inicia mesalazina y decide no reiniciar la terapia biológica, a pesar de la recomendación médica.

Teniendo en cuenta el caso anterior, la EII tiene un impacto en la gestación y el posparto, por lo que, en la práctica clínica, el manejo de este tipo de pacientes es un reto para el gastroenterólogo y ginecólogo. Se presentan múltiples interrogantes a la hora de enfrentarnos a estas dos entidades juntas, las cuales trataremos de responder en el siguiente artículo.

\section{¿CÓMO ES LA RESPUESTA INMUNOLÓGICA NORMAL DURANTE EMBARAZO?}

Los linfocitos T helper (CD4) pueden diferenciarse en dos subtipos de poblaciones con efectos distintos, los Th1 y los
Th2, en función del tipo de citocinas más prevalente en el medio en el que se producen. Si prevalece la IL-12 y el TNF, se favorece la diferenciación a Th1, por el contrario, si lo que más hay en el entorno es IL-4, la vía favorecida es la Th2. Las células Th1 inducen varias acciones citotóxicas e inflamatorias mediadas por la acción de la IL-2, IL-12, INF $\gamma$ y TNF $\alpha$, y son responsables de las reacciones inflamatorias de la inmunidad celular, la hipersensibilidad retardada y la lesión tisular en las enfermedades infecciosas y autoinmunes. Las células Th2 producen, como ya se ha dicho, IL4, IL5, IL6 e IL10, y se asocia con una respuesta de tipo humoral, lo que favorece la aparición de anticuerpos (1).

Hay citocinas que no encajan típicamente con la respuesta Th1 o con la Th2, pero también son importantes en el mantenimiento del embarazo, como son la IL-11 y la IL-18, que están presentes en momentos específicos del embarazo, por lo que sugieren una función reguladora (1). Se ha estudiado que una prevalencia de la respuesta Th1 sobre la Th2 se asocia con tasas mayores de reabsorciones fetales, fallos en la implantación y menor resistencia a la infección y producción de INF $\gamma(2)$. Se ha podido demostrar su acción citotóxica directa sobre el embrión como lesión de células trofoblásticas. Tanto el TNFa como el INF $\gamma$ provocan la inhibición del crecimiento trofoblástico in vitro; sin embargo, las citocinas asociadas con una respuesta Th2 contribuyen a la implantación del embrión, desarrollo de la placenta y supervivencia del feto hasta el término de la gestación (3).

Las distintas citocinas se producen en distinta cuantía según el momento de la gestación en la cual se valoren, así como sus receptores. Tanto las citocinas pro como las antinflamatorias son expresadas en sangre periférica materna, incluida la IL-2, IL-4, IL-10 y el INF $\gamma$, por lo que no parece que, como se pensaba anteriormente, el sistema inmune materno esté comprometido durante el embarazo (4).

La producción en la interfase materno-fetal no solo depende de los relativamente escasos linfocitos T CD4, sino también de otras células productoras de citocinas existentes tanto en el territorio materno como en el fetal. Así, el trofoblasto y los macrófagos en el trofoblasto velloso, así como las células NK, los macrófagos y las células estromales a nivel de decidua contribuyen a mantener este ambiente Th2, que se desarrolla en el transcurso del primer trimestre de gestación. Hacia final del tercer trimestre, y en relación con el parto, se establece una predominancia de tipo Th1, lo que ha llevado a algunos autores a considerar los fenómenos inmunológicos como elementos implicados en los mecanismos de inicio del parto.

Sin que existan datos concluyentes, hay que referenciar el papel de las hormonas en la inmunomodulación durante el embarazo. El estradiol, la progesterona y algunas proteínas placentarias, como la proteína específica del embarazo 
(SP-1), que estimula la producción de citocinas Th2 por los monocitos, y la proteína plasmática asociada con el embarazo (PAPP-A), que inhibe in vitro la proliferación de las células $\mathrm{T}$ y la secreción de IL-2, ayudan a la tolerancia materna al promover la respuesta Th2 sobre la Th1.

Se ha descrito el factor inhibidor de leucocitos (LIF), el cual es sintetizado y secretado por el endometrio materno y células estromales. Su receptor es necesario para la implantación, diferenciación y crecimiento del trofoblasto (1). De acuerdo con lo anterior, entre las sustancias que activan al LIF se encuentran la progesterona, la IL-4 y la IL-1. El INF $\gamma$ y la IL-12 lo inhiben.

La progesterona es necesaria para el mantenimiento del embarazo; para eso, su producción en el cuerpo lúteo es estimulada por las IL-6 y IL-4. Los linfocitos de las mujeres embarazadas son especialmente sensibles a la progesterona. El embarazo por sí mismo aumenta el número de receptores de progesterona que son expuestos en los linfocitos de sangre periférica, haciéndolos más susceptibles de los mecanismos inhibidores de la progesterona. En su presencia, los linfocitos segregan una proteína que tiene un efecto inhibidor directo del efecto citolítico de los NK. Las concentraciones de progesterona son solo para inhibir el sistema inmune localmente en el útero y placenta (1).

En el sincitiotrofoblasto, la progesterona favorece la producción de citocinas Th2. Junto con la progesterona, como ya se ha dicho anteriormente, las catecolaminas y las prostaglandinas inducen la producción de IL-10, así como la gonadotrofina coriónica humana. Los estrógenos, sin embargo, hacen que el endometrio produzca interleucinas quimiotácticas y los macrófagos sean atraídos a la interfase materno-fetal (5). Teniendo en cuenta lo anterior, en el embarazo predomina una respuesta inmune tipo Th2, lo cual podría estar relacionado con el tipo de respuesta desarrollado según el EII.

Un estudio que evalúo los tipos de citocinas en los diferentes EII demostró que la colitis ulcerativa tiende a ser una enfermedad de mayor producción de IL5, lo que conlleva a un predominio de respuesta Th2, lo cual podría tener plausibilidad en la exacerbación de la enfermedad durante la instauración del embarazo.

\section{¿CUÁL ES EL EFECTO DE LA ENFERMEDAD INFLAMATORIA INTESTINAL EN LA FERTILIDAD?}

Las enfermedades inflamatorias intestinales ocurren entre los 33,4 a 45 años, siendo de vital importancia entender que las tasas de infertilidad en mujeres con enfermedad inflamatoria intestinal sin actividad son similares a las de la población general ( $8 \%$ a $10 \%)(6)$. La remisión de la enfermedad no solo mejora las tasas de fertilidad, sino que también, como la mayoría de los estudios han demostrado, conduce a resultados más favorables del embarazo. A su vez, la actividad de la enfermedad favorece la reducción de la fertilidad, probablemente como respuesta al proceso inflamatorio y adherencias que se generan en las trompas de Falopio o los ovarios.

De igual manera, las pacientes que han tenido cirugía previa, como proctocolectomía o anastomosis ileal con reservorio anal, tienen 3 a 4 veces mayor riesgo de infertilidad. Esto debido a las adherencias pélvicas que tienden a formarse, lo que afecta la permeabilidad de las trompas de Falopio y, consecuentemente, conduce a la obstrucción de las mismas (7). Por tanto, el momento adecuado para la concepción es cuando existe remisión de la enfermedad y se recomienda como mínimo 3 a 6 meses de remisión antes de intentar la concepción.

Queda claro que no hay evidencia de que la colitis ulcerativa o la enfermedad de Crohn (EC) inactiva afecten la fertilidad; sin embargo, se ha documentado que las pacientes que con bolsa ileal tienen disminución de la fertilidad.

\section{¿CUÁL ES EL EFECTO DE LA ENFERMEDAD INFLAMATORIA INTESTINAL EN EL EMBARAZO?}

Las pacientes con EII, ya sea EC o CU, tienen peores resultados en el embarazo comparado con mujeres sanas. Los estudios han demostrado, además, que las probabilidades de peores desenlaces del embarazo en pacientes con enfermedad de Crohn son mayores que en pacientes con colitis ulcerativa.

Una revisión realizada en el año 1998 por Subhani y colaboradores encontró que la enfermedad de Crohn, especialmente con actividad de la misma, se asocia con disminución de peso al nacer, parto prematuro y cesárea (8). Además, se evidencia que en las pacientes con EII, la inducción del parto $(32 \%$ vs. $24 \%, p=0,002)$, corioamnionitis $(7 \%$ vs. $3 \%, p=0,04)$ y cesárea ( $32 \%$ vs. $22 \%)$, son más frecuentes que en la población general. Dentro de las complicaciones neonatales, en las cuales se incluyeron bajo peso al nacer, restricción del crecimiento intrauterino, puntuaciones de Apgar y anomalías congénitas, se encontró similitud en poblaciones con EII y sin esta (9). No hay estudios que reporten mayor probabilidad de malformación fetal en estas pacientes, lo cual llama la atención en el caso que exponemos, dado que, si bien pueden existir múltiples factores asociados con malformación fetal, es de notar que el producto de la primera gestación (óbito fetal) se relacionó con el debut de la enfermedad inflamatoria, al presentar las alteraciones morfológicas descritas, lo cual nos invita a reflexionar sobre este tema, y aunque no existe evidencia clara al respecto, sí condiciona que el seguimiento prenatal de estas pacientes sea mucho más estrecho. 
Otro estudio, en el que se incluyó a 461 pacientes embarazadas con EII, demostró que las pacientes con EII tenían más riesgo de presentar un aborto espontáneo, eclampsia, preeclampsia, placenta previa, desprendimiento prematuro de placenta o ruptura prematura de las membranas. En este estudio, la actividad de la enfermedad no se asoció con un resultado peor, sin embargo, se encontró que un diagnóstico de EII, el antecedente de cirugía intestinal por EII y no ser blanco eran predictores independientes de peores resultados (10). Estos resultados soportan las directrices actuales del tratamiento, que indican que el mantenimiento de la remisión durante el embarazo es de vital importancia. Siendo importante saber que el riesgo de este tipo de pacientes podría llegar a ser extrapolable al de la población general si su enfermedad al inicio de la gestación está controlada.

\section{¿CUÁL ES EL EFECTO DEL EMBARAZO EN LA ENFERMEDAD INFLAMATORIA INTESTINAL?}

Aproximadamente, el $80 \%$ de las mujeres con EII que quedan en estado de embarazo cuando la enfermedad se encuentra en remisión tienden a estar en remisión durante todo el embarazo y el período posparto. Se describe que de las pacientes que quedan en estado de embarazo cuando la enfermedad se encuentra activa, cerca del $66 \%$ continúa teniendo una enfermedad activa o presenta empeoramiento de la misma (11). De forma comparativa se describe que hasta el $45 \%$ de las pacientes que tiene diagnóstico de colitis ulcerativa y concibe mientras su enfermedad está activa, esta empeora durante el embarazo, y entre las pacientes con enfermedad de Crohn que conciben mientras su enfermedad está activa, la enfermedad empeora un 30\% (12).

En un estudio prospectivo se encontró que las tasas de exacerbación de la enfermedad fueron similares en pacientes con enfermedad de Crohn embarazadas mientras su enfermedad estaba en remisión y en pacientes con enfermedad de Crohn no embarazadas (3). Por otro lado, las tasas de recaída fueron más altas en mujeres embarazadas con enfermedad de Crohn que concebían mientras su enfermedad estaba activa al compararse con las pacientes no embarazadas con enfermedad de Crohn ( $50 \%$ vs. $33 \%$, respectivamente). Las pacientes con colitis ulcerativa que estuvieron embarazadas tuvieron un mayor riesgo de exacerbación de la enfermedad durante el embarazo y en el período posparto, en comparación con los controles, lo que evidencia que las exacerbaciones de la enfermedad fueron más frecuentes en los primeros 6 meses del embarazo y en los primeros 3 meses del período posparto (13). Por lo anterior debe quedar claro que la actividad de la enfermedad en el momento de la concepción ayuda a predecir el curso de la enfermedad durante el embarazo. Idealmente, las mujeres deben estar en remisión en el momento de la concepción.

\section{¿QUÉ INFLUENCIA TIENEN LOS MEDICAMENTOS UTILIZADOS PARA EL MANEJO DE LA ENFERMEDAD INFLAMATORIA INTESTINAL EN LA FERTILIDAD?}

No existen datos que reporten efectos de los medicamentos en la fertilidad femenina, sin embargo, es de aclarar que el uso de inmunosupresores, como metrotexato, tienen clara asociación con teratogenicidad y están totalmente contraindicados en los pacientes que tienes deseo de concebir.

Se ha descrito que el uso de sulfasalazina condiciona la reducción reversible de la motilidad del esperma, siendo un efecto relacionado con la dosis del medicamento (14). El metotrexato favorece la presencia de oligospermia, que podrá mejorar con el tiempo al suspender el medicamento (15). El infliximab parece afectar la calidad del semen al reducir la motilidad de los espermatozoides (16). Con respecto a la azatioprina no se ha encontrado influencia sobre la calidad del esperma (17).

\section{¿QUÉ EFECTOS ADVERSOS TIENEN LOS MEDICAMENTOS PARA EL MANEJO DE LA ENFERMEDAD INFLAMATORIA INTESTINAL EN EL EMBARAZO?}

De acuerdo con las recomendaciones de las guías de manejo para EII y embarazo, las mujeres que necesitan tratamiento farmacológico para mantener la remisión de la enfermedad deben continuar el uso de los mismos durante el embarazo, teniendo en cuenta que el metotrexato debe ser suspendido previo a la concepción y durante el embarazo. Adicionalmente, si durante el embarazo hay exacerbación de la enfermedad, el tratamiento debe ser agresivo.

Respecto a los medicamentos a utilizar, aunque actualmente ya no es muy utilizada la clasificación de categoría de riesgo en el embarazo según la FDA, describiremos en cual categoría de riesgo están ubicados los medicamentos que frecuentemente se usan en las pacientes embarazadas con EII.

\section{Aminosalicilatos y sulfasalazina (clase B)}

En general se consideran como seguros. En Dinamarca se desarrolló un estudio de cohortes, en el cual se encontró un mayor riesgo de parto prematuro y muerte fetal en mujeres que recibieron aminosalicilatos durante el embarazo; sin embargo, en este estudio no se distinguió entre los efectos de la actividad de la enfermedad y el uso de aminosalicilatos (18). En otros estudios realizados no se encontró asociación significativa entre los aminosalicilatos y efectos adversos durante el embarazo (19).

Es conocido que la sulfasalazina inhibe la síntesis de los folatos, por tanto, las mujeres que usan dicho medicamento deben ser suplementadas con ácido fólico para, de esta 
manera, reducir los efectos adversos sobre el tubo neural (20). En resumen, los aminosalicilatos y la sulfasalazina se pueden usar sin limitación durante el embarazo y no se asocian con resultados adversos significativos.

\section{Tiopurinas (azatioprina) (clase D)}

Se ha evidenciado que este medicamento alcanza niveles séricos fetales tan elevados como el $5 \%$ del nivel del fármaco materno. Los resultados de los estudios en humanos, en términos de seguridad respecto al uso de azatioprina durante el embarazo, han sido discordantes, sin embargo, se recomienda que este medicamento se debe continuar durante el embarazo con el objetivo de mantener la enfermedad en remisión. Estudios recientes han demostrado que el uso de azatioprina no favorece un riesgo mayor para el feto $\mathrm{y}$, a su vez, es seguro continuar el medicamento durante el embarazo, considerándose que la actividad de la enfermedad a cambio del uso del medicamento puede favorecer mayores efectos en el feto (21).

\section{Metotrexato (clase X)}

Es bien sabido que el metotrexato es teratógeno y abortivo, por lo cual está contraindicado durante el tiempo de concepción y embarazo. El uso de metotrexato entre las 6-8 semanas de embarazo puede favorecer la presencia de anomalías congénitas y su uso en el segundo y tercer trimestre favorece la presencia de abortos. Además, se considera que el metrotexato se debe suspender entre 3-6 meses antes de intentar el embarazo, dado que dicho medicamento persiste durante un tiempo activo en los tejidos (20).

\section{Corticoides (clase C)}

Dentro de las características de los glucocorticoides es conocido que atraviesan la placenta y pueden llegar al feto, pero, a su vez, se debe tener en cuenta que las enzimas de la placenta convierten a los corticosteroides en metabolitos menos activos. Este tipo de medicamentos se usan con frecuencia para tratar los episodios de actividad de la enfermedad inflamatoria intestinal. Se encuentran resultados contradictorios en el embarazo, informándose un incremento en la asociación entre los defectos de la fisura orofacial en recién nacidos con el uso de estos en el primer trimestre del embarazo (22).

Existen datos escasos en relación con la dosis exacta de corticosteroides que inducen toxicidad para la madre y el feto, por tanto, la administración de estos se debe realizar con precaución bajo la discreción del médico tratante. Hay estudios extrapolados en otras enfermedades autoinmunes en los cuales se ha documentado que el uso de corticoeste- roides puede favorecer la presencia de parto pretérmino y bajo peso al nacer.

\section{Antibióticos}

Metronidazol (clase B) y ciprofloxacino (clase C) tienen cierta frecuencia de uso en la enfermedad inflamatoria intestinal para tratar abscesos y fístulas. Son medicamentos detectables en leche materna en bajos niveles. En un estudio en mujeres con EII que requirieron uso de metronidazol durante el embarazo se encontró que este era seguro en todos los trimestres; sin embargo, se recomienda evitar su uso en el primer trimestre (23). En lo que respecta a la ciprofloxacina, los estudios no han reportado un incremento significativo de las anomalías congénitas mayores, incluidos los problemas musculoesqueléticos, pero dado el riesgo de artropatía congénita se recomienda no usarla durante el embarazo (24).

En relación con las penicilinas no se ha demostrado que estas condicionen malformaciones fetales o resultados adversos del embarazo y se consideran la primera terapia de línea en el embarazo.

\section{Ciclosporina (clase C)}

Este medicamento atraviesa la placenta, sin encontrarse teratogenicidad en modelos animales. Los estudios realizados con este medicamento están en relación con el trasplante renal, por lo que se sugiere una asociación con bajo peso al nacer y parto pretérmino. De igual manera, en recaídas graves de colitis ulcerativa durante el embarazo, la ciclosporina se ha usado con respuestas favorables al reducir la necesidad de colectomía, sin efectos adversos significativos. El efecto secundario más frecuentemente reportado es la hipertricosis en la madre, otros efectos adversos descritos son nefrotoxicidad y hepatotoxicidad (25). Por lo anterior se puede considerar el uso de ciclosporina en pacientes con colitis ulcerativa fulminante durante el embarazo.

\section{Agentes biológicos (clase B)}

Estos medicamentos incluyen los antiTNF, como infliximab, adalimumab, certolizumab y golimumab. Estos se usan para el manejo de la enfermedad inflamatoria intestinal moderada a severa y en enfermedad de Crohn fistulizante-estenosante. Los niveles de TNF aumentan durante el embarazo, ya que este es principalmente producido por la placenta, siendo importante en las etapas iniciales del embarazo y también para el desarrollo del sistema inmune fetal.

En estudios observacionales y revisiones sistemáticas se ha demostrado la seguridad de su uso durante el embarazo 
(26). En relación con infliximab y adalimumab se debe saber que son anticuerpos monoclonales IgG1 y atraviesan la placenta, mientras que certolizumab es un fragmento $\mathrm{Fab}$ de IgG1, que no tiene transporte transplacentario, por lo cual se ha recomendado no usar infliximab y adalimumab desde el segundo trimestre del embarazo (27). Otros grupos recomiendan continuar la terapia biológica durante todo el embarazo, en especial, en pacientes de alto riesgo y con actividad de la enfermedad, y solo recomiendan su suspensión por deseo materno (28). En caso de considerar suspender la terapia biológica para disminuir la exposición fetal se recomienda detener la administración entre la semana 22 y 26 de la gestación.

No se ha observado aumento en las tasas de aborto espontáneo, muerte fetal, malformaciones congénitas o parto prematuro entre las mujeres embarazadas que han estado expuestas a adalimumab o golimumab; a su vez, los antiTNF no aumentan el riesgo de complicaciones durante el embarazo en comparación con las tiopurinas y el no uso de medicamentos (29).

Niveles de infliximab y adalimumab se han detectado en los lactantes hasta 12 meses luego del parto, sin embargo, no ha existido aumento de las infecciones, reacciones alérgicas o disminución de la respuesta a las vacunas, pero sí se ha observado que los lactantes expuestos a la combinación de inmunomoduladores y agentes biológicos tienen un incremento de infecciones entre los 9 a 12 meses de edad (30).

\section{Antiintegrina (clase C)}

Es un anticuerpo IgG4 monoclonal humanizado que actúa contra la molécula de adhesión a4-integrina, por lo que existen datos limitados sobre el uso de este medicamento durante el embarazo. La revisión de la base de datos de seguridad global de natalizumab no mostró un aumento en los defectos de nacimiento en niños cuyas madres estuvieron expuestas a natalizumab durante el embarazo. En su extrapolación a otras enfermedades se describe en esclerosis múltiple los resultados del embarazo de 35 pacientes que accidentalmente quedaron embarazadas mientras estaban siendo tratados con natalizumab. De estos pacientes, 29 tuvieron embarazos viables, 28 tuvieron niños sin alteraciones y 1 niño nació con hexadactilia. De las 6 pacientes restantes, una decidió someterse a un aborto y las otras 5 tuvieron abortos tempranos (31).

\section{¿CÓMO DEBE SER EL TIPO DE PARTO EN LA EMBARAZADA CON ENFERMEDAD INFLAMATORIA INTESTINAL?}

En el contexto del embarazo y EII se debe tener en cuenta que el parto debe ser dirigido de manera multidisciplinaria en conjunto con el obstetra, gastroenterólogo y coloproctólogo. Ante la presencia de enfermedad activa perianal, compromiso rectovaginal o antecedente quirúrgico de reservorio ileoanal o anastomosis ileorrectal secundaria a EII está indicado el parto por cesárea. Se ha demostrado que el parto vaginal con episiotomía puede estar relacionado con incremento del riesgo de afectación perianal (32). Otro punto por resaltar es que las pacientes con EII sin compromiso perianal tienen indicado el parto vaginal, con todos los beneficios que trae para el neonato. Estudios reportan y sugieren que el parto por cesárea es un factor de riesgo para el desarrollo y exacerbación de EII.

\section{¿QUÉ DEBEMOS TENER EN CUENTA DURANTE LA LACTANCIA?}

La lactancia puede estar asociada con el incremento de la inflamación, ya que la prolactina se relaciona con aumento de la producción del factor de necrosis tumoral, sin embargo, en un estudio realizado no se encontró incremento en la tasa de recaída de la enfermedad en el primer año posparto entre las mujeres que lactaron $(26 \%)$ y las que no lo hicieron $(29,4 \%)$ (33).

Respecto al uso de medicamentos durante la lactancia se debe tener en cuenta que:

- Los aminosalicilatos y sulfasalazina se pueden continuar durante la lactancia, teniendo presente que los aminosalicilatos pueden favorecer la presencia de diarrea osmótica y la sulfasalazina puede condicionar ictericia; sin embargo, las concentraciones del medicamento en leche materna son bajas

- La azatioprina se puede continuar durante la lactancia, esta se detecta en concentraciones bajas de la misma en la leche materna, encontrándose mayores concentraciones del medicamento durante las primeras 4 horas luego del consumo del medicamento, por lo cual, se recomienda desechar la leche obtenida en este rango de tiempo

- El metrotexato, dado su potencial teratogénico, está contraindicado durante la lactancia

- Los corticosteroides se encuentran en concentraciones bajas en la leche materna, encontrándose niveles moderadamente elevados en las primeras 4 horas luego de la toma del medicamento, por lo cual se recomienda desechar la leche obtenida durante este intervalo para reducir riesgo de transferencia al lactante

- Los agentes biológicos se pueden continuar durante el embarazo, encontrándose concentraciones mínimas de infliximab y adalimumab en la leche materna, sin reportarse eventos adversos significativos en el lactante, considerándose que los niveles detectables en el recién nacido después del parto se relacionan con la transferencia placentaria durante el embarazo. Adicionalmente 
no se ha encontrado ninguna asociación entre la lactancia materna y el riesgo de infección en el recién nacido expuesto a agentes biológicos (34).

\section{¿CÓMO DEBE SER LA VACUNACIÓN EN LOS BEBÉS?}

Se debe tener en cuenta que la vacunación con virus no vivos en recién nacidos expuestos a antiTNF en útero no difiere de los bebes no expuestos, por lo que tienen una respuesta a la vacunación adecuada. Con respecto a las vacunas con virus vivos, como rotavirus, polio oral y BCG, debe suministrarse cuando los niveles de antiTNF no sean detectables, por lo cual, no deben recibir vacunas vivas durante los primeros 6 meses de vida, por tanto, se realiza la recomendación de la suspensión de los antiTNF a la semana 33 de embarazo o, como algunos otros autores lo sugieren, desde la semana 26 , de esta manera se puede llegar al momento del parto con niveles indetectables del mismo, lo cual favorece la no afectación del esquema de vacunación del neonato.

\section{CONCLUSIÓN}

El equipo médico que se encuentra involucrado en el seguimiento de las pacientes con EII debe saber enfrentarse y tener claridad en el manejo preconcepcional, concepcional y posparto de estos pacientes, ya que se deben tener en cuenta múltiples factores, como lo son el control de la enfermedad durante la época fértil, para poderse llevar a cabo un embarazo en buen término $y$, a su vez, se debe tener claridad acerca del uso de los medicamentos durante las distintas etapas en las que se encuentra la mujer en edad concepcional, lo cual generará tranquilidad tanto para el médico tratante como para la paciente; por tanto, dicha sensibilización de una educación apropiada favorece el incremento de la probabilidad de que los médicos sigan pautas para mejores prácticas en el manejo de pacientes embarazadas con EII.

\section{Conflicto de interés}

No hubo conflicto de interés.

\section{Fuente de financiación}

No hubo fuente de financiación.

\section{REFERENCIAS}

1. Lewis A. Why isn't the foetus rejected? A study of the changes that occur in the mammalian immune system to allow for a successful pregnancy, Faculty of Health and Biosciences. Stratford, London.

2. Kanellopoulos-Langevin C, Caucheteux S, Verbeke P, Ojcius DM. Tolerance of the fetus by the maternal immune system: role of inflammatory mediators at the feto-maternal interface. Reprod Biol Endocrinol. 2003;2;1:121. doi: https://doi.org/10.1186/1477-7827-1-121.

3. Dealtry G, O'Farrell MK, Fernandez N. The Th2 cytokine environment of the placenta. Int Arch Allergy Immunol. 2000;123(2):107-19. doi: 10.1159/000024441.

4. Hanna N, Hanna I, Hleb M, Wagner E, Dougherty J, Balkundi D, et al. Gestational age-dependent expression of IL-10 and its receptor in human placental tissues and isolated cytotrophoblasts. J Immunol. 2000;164(11):5721-8. doi: https://doi.org/10.4049/jimmunol.164.11.5721.

5. Ozenci C, Korgun ET, Demir R. Immunohistochemical detection of CD45+, CD56+, and CD14+ cells in human decidua during early pregnancy. Early Pregnancy. 2001;5(3):164-75.

6. Steinlauf A, Present D. Medical management of the pregnant patient with inflammatory bowel disease. Gastroenterol Clin North Am. 2004;33:361-85. doi: https://doi. org/10.1016/j.gtc.2004.02.013.

7. Gorgun E, Remzi F, Goldberg JM, Thornton J, Bast J, Hull T, et al. Fertility is reduced after restorative proctocolectomy with ileal pouch anastomosis: a study of 300 patients. Surgery. 2004;136:795-803. doi: https://doi. org/10.1016/j.surg.2004.06.018.

8. Subhani J, Hamilton M. El manejo de la enfermedad inflamatoria intestinal durante el embarazo. Aliment Pharmacol Ther. 1998;12:1039-533.

9. Bush M, Patel S, Lapinski R, Stone J. Resultados perinatales en la enfermedad inflamatoria intestinal. J Matern Fetal Neonatal Med. 2004;15:237-41. doi: https://doi.org/10.10 $80 / 14767050410001668662$.

10. Mahadevan U, Sandborn W, Li D, Hakimian S, Kane S, Corley D. Pregnancy outcomes in women with inflammatory bowel disease: a large community-based study from Northern California. Gastroenterology. 2007;133(4):110612. doi: https://doi.org/10.1053/j.gastro.2007.07.019.

11. Mogadam M, Korelitz B, Ahmed S, Dobbins W, Baiocco P. El curso de la enfermedad inflamatoria intestinal durante el embarazo y el posparto. Am J Gastroenterol. 1981;75(4):265-9.

12. Miller J. Enfermedad inflamatoria intestinal en el embarazo: una revisión. JR Soc Med. 1986;79(4):221-5. doi: https:// doi.org/10.1177/014107688607900410.

13. Pedersen N, Bortoli A, Duricova D, D’Inca R, Panelli M, Gisbert J, et al. The course of inflammatory bowel disease during pregnancy and postpartum: a prospective European ECCO-EpiCom study of 209 pregnant women. Aliment Pharmacol Ther. 2013;38(5):501-51. doi: https://doi. org/10.1111/apt.12412.

14. Levi A, Fisher A, Hughes L, Hendry W. Male infertility due to sulphasalazine. Lancet. 1979;314(8137):P276-8. doi: https://doi.org/10.1016/S0140-6736(79)90292-7. 
15. Sussman A, Leonard J. Psoriasis, methotrexate, and oligopsermia. Arch Dermatol. 1980;116:215-7. doi: 10.1001/ archderm.1980.01640260091025.

16. Mahadevan U, Terdiman J, Aron J, Jacobsohn S, Turek P. Infliximab and semen quality in men with inflammatory bowel disease. Inflamm Bowel Dis. 2005;11(4):395-9. doi: https://doi.org/10.1097/01.MIB.0000164023.10848.c4.

17. Dejaco C, Mittermaier C, Reinisch W, Gasche C, Waldhoer $\mathrm{T}$, Strohmer $\mathrm{H}$, et al. Azathioprine treatment and male fertility in inflammatory bowel disease. Gastroenterology. 2001;121(5):1048-53. doi: https://doi.org/10.1053/ gast.2001.28692.

18. Nørgård B, Puho E, Pedersen L, Czeizel A, Sørensen HT. Risk of congenital abnormalities in children born to women with ulcerative colitis: a population-based, case-control study. Am J Gastroenterol. 2003;98:2006-10. doi: https:// doi.org/10.1016/S0002-9270(03)00427-1.

19. Moskovitz D, Bodian C, Chapman M, Marion J, Rubin P, Scherl E, et al. The effect on the fetus of medications used to treat pregnant inflammatory bowel-disease patients. Am J Gastroenterol. 2004;99:656-61. doi: https://doi. org/10.1111/j.1572-0241.2004.04140.x.

20. Nielsen O, Maxwell C, Hendel J. IBD medications during pregnancy and lactation. Nat Rev Gastroenterol Hepatol. 2014;11:116-27. doi: https://doi.org/10.1038/nrgastro.2013.135.

21. Polifka J, Friedman J. Teratogen update: azathioprine and 6-mercaptopurine. Teratology. 2002;65(5):240-61. doi: https://doi.org/10.1002/tera.10043.

22. Carmichael S, Shaw G. Maternal corticosteroid use and risk of selected congenital anomalies. Am J Med Genet. 1999;86(3):242-4. doi: https://doi.org/10.1002/ (SICI) $1096-8628$ ( 19990917$) 86: 3<242:$ : AID AJMG9>3.0.CO;2-U.

23. Koss C, Baras D, Lane S, Aubry R, Marcus M, Markowitz $\mathrm{L}$, et al. Investigation of metronidazole use during pregnancy and adverse birth outcomes. Antimicrob Agents Chemother. 2012;56:4800-5. doi: https://doi. org/10.1128/AAC.06477-11.

24. Nahum G, Uhl K, Kennedy D. Antibiotic use in pregnancy and lactation: what is and is not known about teratogenic and toxic risks. Obstet Gynecol. 2006;107:1120-38. doi: https://doi.org/10.1097/01.AOG.0000216197.26783.b5.
25. Paziana K, Del Monaco M, Cardonick E, Moritz M, Keller $\mathrm{M}$, Smith B, et al. Ciclosporin use during pregnancy. Drug Saf. 2013;36:279-94. doi: https://doi.org/10.1007/ s40264-013-0034-x.

26. Schnitzler F, Fidder H, Ferrante M, Ballet V, Noman M, van Assche $G$, et al. Outcome of pregnancy in women with inflammatory bowel disease treated with antitumor necrosis factor therapy. Inflamm Bowel Dis. 2011;17:1846-54. doi: https://doi.org/10.1002/ibd.21583.

27. Gisbert J, Chaparro M. Safety of anti-TNF agents during pregnancy and breastfeeding in women with inflammatory bowel disease. Am J Gastroenterol. 2013;108:1426-38. doi: https://doi.org/10.1038/ajg.2013.171.

28. Dubinsky M, Abraham B, Mahadevan U. Management of the pregnant IBD patient. Inflamm Bowel Dis. 2008;14(12):1736-50. doi: https://doi.org/10.1002/ ibd.20532.

29. Djokanovic N, Klieger-Grossmann C, Pupco A, Koren G. Safety of infliximab use during pregnancy. Reprod Toxicol. 2011;32:93-7. doi: https://doi.org/10.1016/j.reprotox.2011.05.009.

30. Hellwig K, Haghikia A, Gold R. Pregnancy and natalizumab: results of an observational study in 35 accidental pregnancies during natalizumab treatment. Mult Scler. 2011;17(8):95863. doi: https://doi.org/10.1177/1352458511401944.

31. Brandt L, Estabrook S, Reinus J. Results of a survey to evaluate whether vaginal delivery and episiotomy lead to perineal involvement in women with Crohn's disease. Am J Gastroenterol. 1995;90:1918-22.

32. Moffatt D, Ilnyckyj A, Bernstein C. A population-based study of breastfeeding in inflammatory bowel disease: initiation, duration, and effect on disease in the postpartum period. Am J Gastroenterol. 2009; 104:2517-23. doi: 10.1038/ajg.2009.362.

33. Grosen A, Julsgaard M, Kelsen J, Christensen L. Infliximab concentrations in the milk of nursing mothers with inflammatory bowel disease. J Crohns Colitis. 2014;8:175-6. doi: https://doi.org/10.1016/j.crohns.2013.09.003.

34. Nguyen G, Seow C, Maxwell C, Huang V, Leung Y, Jones J, et al. The Toronto Consensus Statements for the Management of Inflammatory Bowel Disease in Pregnancy. Gastroenterology. 2016;150:734-57. doi: https://doi. org/10.1053/j.gastro.2015.12.003. 